\title{
A multi-case study on Industry 4.0 for SME's in Brandenburg, Germany
}

\author{
Mayur Andulkar \\ BTU Cottbus-Senftenberg \\ $\underline{\text { mayur.andulkar@b-tu.de }}$
}

\author{
Duc Tho Le \\ BTU Cottbus-Senftenberg \\ ductho.le@b-tu.de
}

\author{
Ulrich Berger \\ BTU Cottbus-Senftenberg \\ ulrich.berger@b-tu.de
}

\begin{abstract}
Industry 4.0 is still in its development phase and it promises to bring remarkable benefits to the manufacturing industry around the world when employing the Smart Factory application in large organizations and their supply chains. However, there is a risk of a miss-match when trying to introduce Industry 4.0 to Small and Medium Enterprises (SME) as the concept is mainly being developed around large manufacturing companies. The purpose of this research is to analyze the readiness level and feasibility of implementing Industry 4.0 technologies for SME's in the federal state of Brandenburg (Germany). The work is based on the survey of 20 SME's assessing their current problems emphasizing on automation, Enterprise Resource Planning (ERP), CAD/CAM, factory layout planning and logistics. Five SME's from different domains out of the 20 surveyed are taken as case studies to evaluate the potential benefits, trade-offs and barriers from an implementation of these integrated technologies. The findings revealed that the companies are still coping with the issues relating to planning, logistics and automation. It was also found that all the concepts of $i 4.0$ may not be necessary or even beneficial to an enterprise in the current scenario and new strategies need to be developed for its realization in SME's.
\end{abstract}

\section{Introduction}

The manufacturing industry is undergoing a huge transformation because of several factors such as globalization, urbanization, individualization, and demographic change which will considerably challenge the entire manufacturing environment in the future [1]. These challenging environments will force the companies to adapt themselves by changing their structure, processes or products.

There has been a need to address these challenges and strengthen the competitiveness of the manufacturing industry in developed countries (such as US, Germany, Japan etc.), where this sector accounts for more than $10 \%$ of their GDP [2][3]. Among these countries, the developments in Germany, which has been Europe's industrial powerhouse and the world's second largest exporter, have gathered larger attention from the world. It is not just because Germany has many large multinational companies but also the SMEs in the country are suppliers to several multinational corporations across the world.

One of the key developments in Germany was in 2011 when the German federal government announced the $4^{\text {th }}$ revolution in industry (Industry 4.0) as one of the key initiatives of its high-tech strategy [4]. However, unlike previous revolutions in industry, Industry 4.0 (i4.0) is predicted a-priori and not ex-post [5]. Thus, there are only anticipations and predictions regarding the concepts and technologies in i4.0 with far reaching effects ranging from increase in the operational effectiveness to the development of entirely new business models, services, and products.

Although these concepts and technologies of i4.0 are still under development, there is a risk that they are being developed taking into consideration only the large manufacturing companies, for instance the automotive industry, rather than being more generally designed. This could potentially endanger the SME sector which generally forms the backbone of most economies. For instance, in Germany, the SME sector accounts for $99.5 \%$ of all organizations (that is more than 3.6 million companies) including the manufacturing, trade, services and construction industries and employing about $62.8 \%$ of the German work force [6]. Thus, the success of i4.0 and approaches under it will depend on whether the SMEs can adopt and implement these technologies. Moreover, if these concepts could only be used by the large manufacturing enterprises (owing to the complexity and required expertise in the underlying technologies) there is a high risk of a mismatch which may very well endanger the very existence of the SMEs. Sommer et al. [7] explains the future of SMEs and how excluding them from i4.0 concept could have huge negative impact on the German economy. Consequently, a successful implementation 
of i4.0 is also highly linked to the capability of SMEs facing and adapting to this change [8].

Since i4.0 is likely to affect different industries across several sectors, studies related to its implementation have been conducted by several researchers across the world. Faller et al. [9] present the learning factory for bringing i4.0 to SMEs; the findings of Armin Decker [10] over the state of SMEs in Jutland Region of Denmark reveal that the SMEs need to overcome significant hurdles to be successful players in future i4.0 developments. Lutz Sommer [7] gives the results of nine studies dealing in the range of topics related to i4.0 and finds that smaller SMEs might become victims instead of beneficiaries of the revolution. These findings suggest that there is a huge gap between the concepts and technologies proposed under i4.0 and the current state of SMEs.

As the concept of i4.0 promises huge gains to the companies that adopt these technologies and at the same time considering the existing gap between these concepts and their application to the SMEs, this paper focuses on the evaluation of the readiness level of SMEs for implementation of the concepts proposed under i4.0 for SMEs in the federal state of Brandenburg, Germany. A qualitative survey of SMEs in Brandenburg was conducted to identify their current problems and understand how the implementation of the concepts under i4.0 could benefit them. In the survey, 20 SMEs were visited and analysed for their current problems and readiness level for i4.0 technologies in their current state. As the 20 SME's surveyed were in different domains with different objectives and variety of products, it was difficult to categorize them in one group for evaluation on concepts of i4.0. Also, the implementation of these concepts required consideration of several factors including the size of enterprise, domain, existing processes and infrastructure etc. Hence, in this paper, five SME's from different industry sectors were taken as case studies, analyzed for their readiness level and potential benefits, trade-offs and barriers from implementing i 4.0 concepts.

The rest of the paper is structured as follows. Section 2 discusses the four core technologies in i4.0. Section 3 details the results of the survey conducted and mentions the current problems of SME's in Brandenburg. In Section 4 five case studies in implementing i4.0 technologies mentioned in Section 2 are taken and an analysis of the same is performed. A discussion about the case studies is presented in Section 5 and finally conclusion and outlook is presented in Section 6.

\section{Technologies in Industry 4.0}

The concept of i4.0 originated in Germany and has gained momentum in recent years building high expectations around its outcomes. It addresses the competition of low-cost labor resources faced by the companies in developed countries by reducing the overheads of low-skilled labor [8]. There have been several definitions of $\mathrm{i} 4.0$ and confusion related to the underlying concepts. Hermann et al. [11] and Roblek et al. [12] proposed i4.0 with four technology concepts as shown in Fig. 1 which has been followed and used extensively in several studies. This framework has been followed in this work and the underlying concepts are discussed as follows.

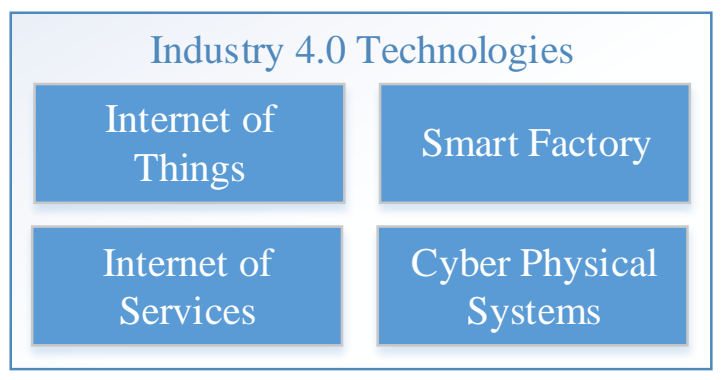

Figure 1: Technologies in i4.0

\subsection{Internet of Things (IoT)}

The IoT is an inter-networking of 'things' and 'objects', such as RFID, sensors, actuators, mobile phones that interact and co-operate with each other to reach common goals [13]. It enables the ability to combine physical and digital components (or software) in order to create new ones resulting in smart products [14] (for example smart transport, smart cities, smart factories and so forth). IoT for industrial purposes is different from the user based IoT due to demand for real time data availability and high reliability. Thus, IoT applied to industrial processes is referred as Industrial Internet of Things (IIoT). IIoT offers product traceability throughout the entire product lifecycle and enables flexibility and operational efficiencies, reshaping the supply chain and manufacturing process. Typical applications of IIoT in industry are predictive maintenance, remote asset management, improvement of worker productivity, safety and working conditions and differentiated customer experiences [15].

\subsection{Internet of Services (IoS)}

The manufacturing industry which has conventionally been product-oriented has shifted to 
service-oriented manufacturing [16] as it enables gaining revenue from service transactions all along the life cycle of a product service system (PSS) [17]. The shift to service-oriented architecture enables high product quality and at the same time the value-added services give the companies an appropriate opportunity to differentiate themselves ensuring a strong competitive position. This has led to the development of IoS which is an infrastructure that uses the Internet as a medium for offering and selling services and making them tradable [18]. Through IoS the data of a product can be acquired even during its operation and used for the development of new services and updates consequently increasing the perceived product quality. In this paper, the term Internet of Services is considered as the technology that monitors the product life cycle, taking decisions based on data gathered through the product life for predictive maintenance, seamless production flow and reliability of machines and products.

\subsection{Cyber Physical System (CPS)}

CPS's are a fusion of cyber world and dynamic physical world with integrated computational and physical capabilities to interact with the environment through several modalities. They are characterized by a network of interacting elements where sensors (cyber objects) can be used to monitor the physical environments, and the actuators/controllers can be used to change the physical parameters [8]. When compared to the Internet which is based on the integration of network technology, applications and infrastructure; CPS's can be seen as the integration of embedded systems, sensors, and control systems [19]. Examples of CPS include biomedical and healthcare systems, smart grids, autonomous vehicles etc.

There are several architectures proposed for CPS as detailed in [20] most of which are developed considering service-oriented architecture due to the shift to service-oriented manufacturing. The 5-level CPS structure proposed by Lee et al. [21] is adopted in this paper as it provides guidelines for developing and deploying a CPS for manufacturing applications.

\subsection{Smart Factory}

The development of IoT, IoS and CPS has led to the possibility of a smart factory which is highly flexible, reconfigurable, capable of producing customized products and small-lot products efficiently and profitably [22]. Just like humans live in two worlds i.e. physical world and cyber (internet) world, the factory will co-exist in two worlds: physical world and a digital twin in the cyberspace.
The digital twin will take the data generated from sensor networks and manual inputs, process the data in cyberspace and take corrective actions in real time to effect the physical world [23]. The smart factory framework for i4.0 proposed by Wang et al. [22] is adopted in this paper which consists of four tangible layers, namely, physical resource layer, industrial network layer, cloud layer, and supervision and control terminal layer.

The evaluation of SME's based on the above four concepts is performed in the next section.

\section{Current problems with SME's in Brandenburg}

As seen in previous section, the technologies under i4.0 promise a huge advantage to the companies in every aspect. To evaluate this in the state of Brandenburg, a survey of 20 SMEs was conducted. The survey was conceptualized based on the response of SMEs, the requirements for solving their current problems and experience with previous projects in the domain. Initially, a questionnaire was sent to the companies which included questions regarding the company profile, its technical status and organizational details, strategy for the future and current problems faced by the company. Based on the response of questionnaire, an analysis of the company was performed. Next, a visit to the company was conducted and detailed discussions with the director, production or technical managers or other responsible employees were conducted to provide more complete information for the analysis. The discussions during the visit were particularly useful as they provided a direct insight into the company's organizational structure and the know how about the available technologies. The discussions were mainly conducted with the company director $(54 \%)$, followed by production managers (23\%), marketing and sales management personnel (12\%) and technical managers (11\%) as shown in Fig 2.

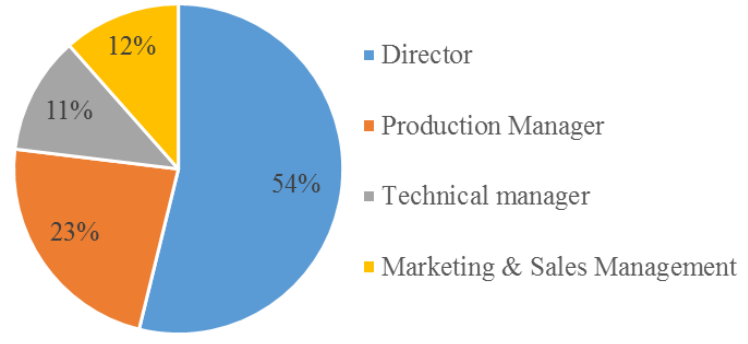

Figure 2: Role of the personnel in the discussions conducted on-site 
Based on the discussions conducted on-site, an analysis of the current problems faced by the companies was performed and an evaluation of the solutions that i4.0 technologies offer was conducted.

The companies that were surveyed have been clustered into micro- ( $<10$ employees), small- ( $<50$ employees) and medium-sized enterprises $(<250$ employees) in accordance with the European Commission (2003/361/EC of 6 May 2003) [24]. As can be seen from Fig.3, majority of the surveyed enterprises were small-sized $(60 \%)$ enterprises followed by medium-sized enterprises with $30 \%$ and only two of the surveyed companies were microsized. The different industry fields for the surveyed enterprises are visible from Fig. 3. The strongest represented areas in the surveyed enterprises are the steel and metal processing $(50 \%)$ followed by manufacturing (20\%), closely followed by the services industry $(15 \%)$.

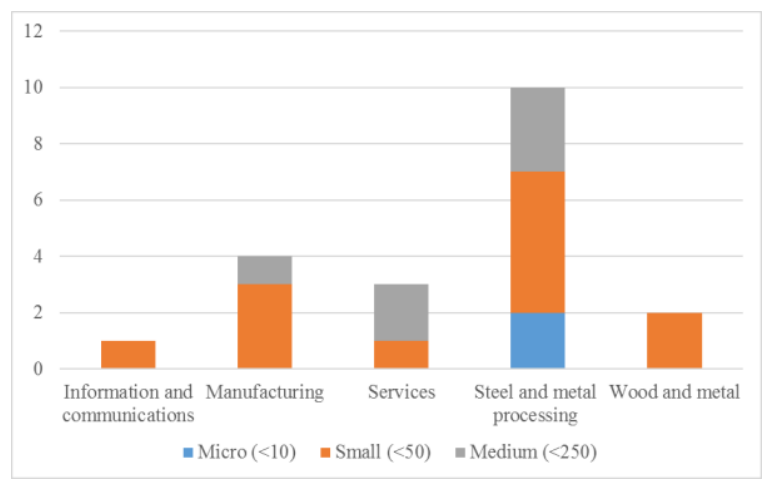

Figure 3: Industry sectors and classification of the surveyed companies

Each enterprise visited was surveyed to evaluate existing problems related to different domains as shown in Fig. 4. Most of the companies faced problems in the domain of Logistics and Supply Chain Management (SCM) (60\%), Factory layout Planning (50\%), Production Planning and control Systems (PPS) (50\%), Automation (50\%) and Enterprise Resource Planning System (ERP) (40\%). It can also be seen that few companies faced problems with digitalization and CAD/CAM. As digitalization is a key feature for companies to adapt Industry 4.0 concepts, it could be feasible for the companies to implement the technologies of IoT, IoS, CPS and Smart Factory as discussed in Section 2.

As shown in Fig 5. all the enterprises were found to have problems with Logistics and SCM. The Steel and metal processing industries and manufacturing industries were struggling with the problems in almost all the domains.

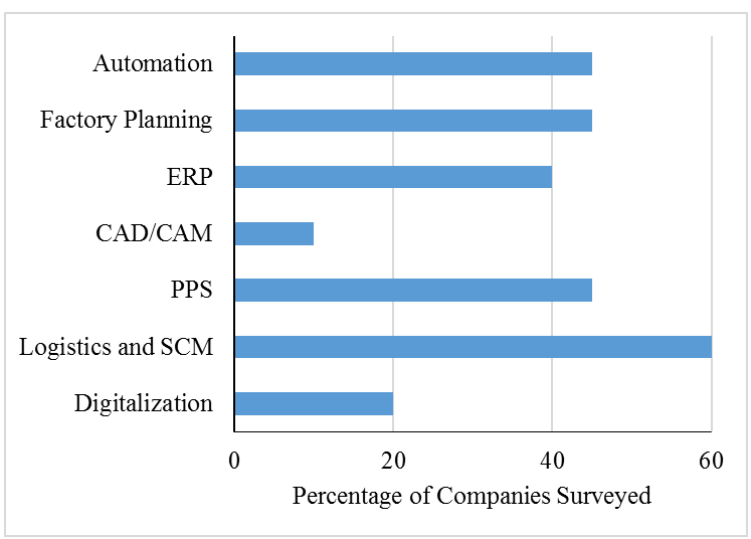

Figure 4. Summary of problems with SME's in Brandenburg

Next, from the above surveyed companies, five case studies of SME's from different sectors were taken as use cases and the implementation of i4.0 technologies in their current state was analysed and the potential benefits, trade-offs and barriers from their implementation was analysed. It is pointed out to the reader that the scope of the paper is limited to the company, its needs and resources available in the company. The aspects of merger or integration with other businesses are not considered here.

\section{Case Studies}

The following section presents case studies in implementing i4.0 concepts for SME's based on the material collected from the survey and observations in the company. The names of companies are kept confidential and are written alphabetically.

\subsection{Company A: Steel and Metal Processing SME}

Company A is active in steel and metal construction and mainly manufactures components for power plants. It is a small enterprise $(<50$ employees) and primarily serves customers within Germany. 


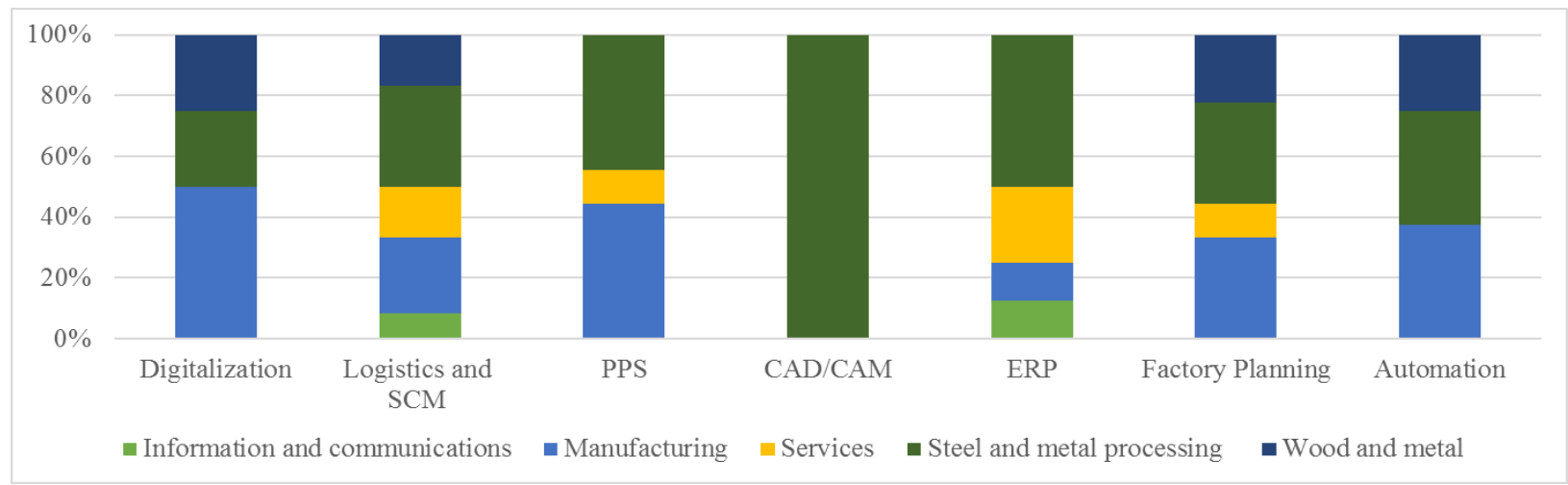

Figure 5: Domain specific breakout of the problems faced by SMEs

The company has cutting machines as well as several welding sites, where very large parts are joined together. The workstations are currently distributed in two manufacturing halls. Consequently, an efficient distribution of the workstations is planned for the optimization of the production routes.

The order planning is currently carried out using Excel lists, since the existing merchandise management system does not include production planning. The planning is characterized by a short forecast, a high variety of variants as well as the integration of the external galvanizing plant. As the Excel lists are maintained by several people, it is impossible to ensure availability of up-to-date data.

There are several storage areas in production. For technical and economic reasons, parts are sometimes produced beyond demand. Frequently these should be searched since no fixed storage locations are defined. The aim is to mark the finished parts, which can still be identified even after installation at the customer location, to support the maintenance teams of another company.

\section{Analysis}

Regarding the technologies used in the organization and compared to the concepts of i4.0 as discussed in Section 2, it was found that the company uses computer controlled machines for manufacturing and documentation is done digitally. However, with regards to the concept IoT, the co-ordination between different components was missing. The company is adopting to concept of $\operatorname{IoS}$ with an initial step of marking the product through its life cycle. The company does not use data analytics to predict the problems or for system monitoring. With regards to the concept of smart factory, a digital twin is unavailable and data is generated from the machines is not connected to the cyber world. As the company has computer controlled machines, there is a possibility to upgrade them to CPS but that would require investments and the CPS should be tailored to the company demands.

\section{Identified potential benefits, trade-offs and barriers}

The implementation of IoT and IoS concepts is difficult sin the current situation but could be implemented for targeted processes. With regards to the current problems, it would enable the customer orders to be transferred to production orders through ERP or existing merchandise management system via tracking technology. This would also enable the actual status of the production order to be tracked for relevant processing steps resulting in less delays and quasistandardization of the production process. Moreover, a machine assignment plan can be generated by integrating the orders planned by the management. The optimum machine utilization could also be possible allowing new orders to be planned better, which leads to better delivery times and reduction of idle time. A step in the direction of smart factory could be achieved by initially simulating the production process. This would help in the factory set-up and bottlenecks and optimization possibilities to be detected at an early stage.

The current problem of storage at fixed locations could be addressed through dynamic storage allowing the optimization and utilization of the storage areas and the routes. IoT could assist in recording the location of all parts, helping in flexible and quick retrieval of components.

\subsection{Company B: Wood and furniture SME}

The company is a small owner-managed enterprise (<50 employees) specialized in the production of highquality picture frames made of wood, plastic and aluminum according to customer requirements. It aims to make the highest percentage of sales through online sales in Germany. The online shop is the main distribution channel for the customer service and $5 \%$ of the sales are still made manually. 
The orders are generally accepted electronically (via the online shop and via e-mails). The production process is carried out by means of an order booklet, which tracks the products along the various workstations. The product range is characterized by a high number of variants (profile, color, size, 6 types of glass) a fact which makes the digital tracking of the production processes more difficult.

An order is first processed by the administrative staff; the order handling slip being prepared with the appropriate priority. In the next step, the bill of material is created, whereby, in the case of material requirements, a purchasing order for bars, glass, auxiliary materials etc. is triggered. The actual production takes place after goods receipt or picking.

The manufacturing process comprises the following workstations: cutting, stitching, completing, welding, packing and shipping. The number of orders varies on a daily basis. The company is looking to expand the product range through new sales channels.

Analysis:

It was observed that the company has already infrastructure for IoT and IoS partly. The order placement is performed through the system online however, order tracking and prediction is not performed. The manufacturing process involves a lot of manual intervention and could be integrated with the existing infrastructure to the cyber world through a merchandise management system. The concept of CPS and smart factory in this context is very much relevant as the part orders are highly variable with several variants. However, the existing infrastructure is not being utilized optimally to implement this.

\section{Identified potential benefits, trade-offs and barriers}

Due to the availability of existing infrastructure, it would be easier to implement IoT concepts in different processes. The concept of IoT could be leveraged here for order tracking and to realize a foresight of the production and delivery time. The existing ERP system could be supplemented through order tracking resulting in reduced the search times, appropriate delivery times, and establishment of production process standardization. The IoT would also determine current occupancy of the workstations and predict its future utilization for production. The connection of the office and production network would help in optimum utilization of resources and a better coordination between company management / work preparation and production. There is not a lot of scope for shifting to service-oriented architecture and hence less IoS possibilities. A smart factory with a digital twin would enable low downtime and high throughput. However, its implementation would require high investments and expertise.

\subsection{Company C: Services}

The company is a micro ( $<10$ employees) ownerrun craft company. The company specializes in the manufacture and installation of vehicle loader facilities according to customer requirements. The vehicles are fitted with shelf and cabinet systems according as desired. The orders are taken personally and cabinets systems are designed manually as required. The cabinets are manufactured manually with tools by experts where the planning software is used to generate the bill of materials. The installation of the cabinets is done manually in the vehicle.

\section{Analysis:}

The company takes orders personally due to the customized nature of requirements. There is limited use of automated machines and components related to IoT, IoS, CPS or smart factory. Due to the highly customized requirements, it is difficult and complex to standardize the procedures and methods for the products. It was realized that the infrastructure for implementing the concepts of i4.0 on such a micro level may not be applicable and the technologies for such applications are still under development.

\section{Identified potential benefits, trade-offs and barriers}

The use of internet for order placement in this application may not be feasible due to the customized requirements of the user and the possibility of including these customizations into the product. However, the company could use the data from the previous orders to standardize their components, predicting the future orders and for stock optimization. But the concept of IoT and IoS in its totality would require huge investment and efforts which are not feasible for this company. The concept of CPS and smart factory are less relevant for this company as there is limited level of automation and most of the work is required to be performed manually. The company first needs standardization and production planning for efficient utilization of resources and cost reduction.

\subsection{Company D: Manufacturing SME}

The company is a small enterprise ( $<50$ employees) and specializes in manufacturing of roller shutters with plastic and aluminum armor, sun and insect protection, the latter being produced seasonally. It is manufactured exclusively according to customer requirements (or customer order) for commercial customers, who realize 
delivery and installation at the end customer. The tanks are manufactured in the main company's manufacturing and the finished roller shutters are assembled.

Due to customer-oriented production, there is a high number of variants (in color, drive, dimensions, etc.) and varying order quantities. In some cases, changes are made by the customer after production start. The orders are received digitally and the order processing and tracking is done by the ERP but the manufacturing process uses hard copies for production plans. Due to several variants, many different input materials are required to meet the short delivery times expected by the customer.

\section{Analysis}

From the i4.0 concepts, it was found that the company performs order processing and tracking digitally. Thus, the infrastructure for IoT concepts is available. There is a possibility for IoS via a shift to service-oriented manufacturing through product life cycle management and value-added services. The production process and logistics involves tracking stations monitored through ERP but is not optimized for time and resources. The company does not use data analytics to predict the problems or for system monitoring. With regards to the concept of CPS, the machines used for production are computer controlled but not connected through cyber space. For a smart factory implementation, a digital twin is unavailable and the initial steps in this direction are being taken by performing simulations of the production process.

\section{Identified potential benefits, trade-offs and barriers}

Implementing IoT would vastly improve the productivity of the company allowing for comprehensive and continuous order processing. The order tracking could also be optimized with removal of data duplication and redundant tracking stations. Through IoT the current processing status, processing times as well as the exact material consumption can be recorded and evaluated for each step of the process via the confirmation of the process. As a result, the production process would become more transparent and interim and post-costing as well as short-term customer-specific changes for orders would be possible at any time helping in future order prediction. The data available from material consumption for individual orders would help in inventory optimization (safety stocks, procurement strategies, etc.).

The implementation of IoS would need a change in business strategy with high investments and long-term plan. IoS could possibly benefit the company by generating revenue through services and also help improve the product quality and competitiveness in the market.

With regards to CPS, the company can start with Smart Connection level (Level 1) [21] for making a sensor network and infrastructure for analytics. This would require significant investments and effects of CPS would only be visible in longer run.

The company has taken initial steps in the direction of smart factory by simulating its production processes and material flows. This would result in optimization of different processes before they are implemented and bottlenecks in production could be identified.

\subsection{Company E: Manufacturing SME dealing in Plastics}

The company is a small enterprise (<50 employees) engaged in the milling of drawing-bonded plastic moldings in micro, small and medium series. The main customers are automotive suppliers, packaging machine manufacturers and manufacturers of medical devices, mostly from Germany. The orders are accepted telephonically and through email.

After the order has been issued by the customer, the order is planned centrally by means of the Manufacturing Execution System (MES) integrated into the ERP, however the warehouse is managed via Excel. The current and planned capacity utilization of the machines as well as the current order status can be called up at any time via the MES. The confirmation of the orders is made via the production data acquisition terminals in the production department.

For manufacturing, the company has several $\mathrm{CNC}$ milling centers for machining of plastic parts. Due to the nature of plastic material, there can be higher feed rate with tool wear significantly lower compared to the metal working. However, due to the more frequent tool changes, other system components wear out significantly faster. In addition, the processing times of the components are very low, so that the machine setup time plays a central role in the processing of the orders.

\section{Analysis}

As compared to the concept of IoT the company does have an online portal for order placement and warehouse management. For the resource management, the company uses ERP software and there exists a communication network between management and production sides. The company could use the existing infrastructure for data processing and analysis which will help in order prediction and resource optimization.

The company could shift towards service oriented architecture and leverage the advantages of IoS and 
implement product life cycle management to improve the quality of parts produced.

The concepts of CPS and smart factory are relevant in this company as they can provide flexibility to address the problem of high variation in parts and small batch sizes. With regards to CPS, the company is in the initial phase of Smart Connection level (Level 1) [21].

\section{Identified potential benefits, trade-offs and barriers}

From the analysis, implementing IoT would vastly assist the company in improving over several aspects. The use IoS would assist the company but it would require investments in terms of time, money and a change in the company strategy. The company has taken initial steps in the direction of CPS by implementing an ERP and MES to track and manage the production system. The existing infrastructure could be used for data processing and decision making. With regards to the smart factory concept, the simulation of the production process is not yet performed. Initial steps in this direction would help the company optimize its resources and reduce operating costs. This could also allow new orders to be scheduled more efficiently leading to shortening of processing times.

\section{Discussion on feasibility of implementing Industry 4.0}

From the case study analysis, it is evident that a significant part of the technologies necessary for industry 4.0 are still in their initial phase, that is, there are still considerable requirements to be fulfilled. The existing frameworks for implementing $\mathrm{i} 4.0$ in companies such as 5-level structure for CPS [21] or the smart factory framework for i4.0 [22] are not sufficient to evaluate the readiness of the SMEs. In many cases, these frameworks have basic requirements which are also not fulfilled or are in the initial stages. For example, for Company A, a communication network is not yet implemented and it would still require significant efforts and investments. Moreover, several i4.0 technologies are still under development and it is challenging for small and medium-sized enterprises to dedicate resources for these technologies. They would in several cases prefer to use these technologies as offthe-shelf products (instead of developing in-house) to achieve product innovation.

These findings agree with the data from Federal Statistical Office of Germany [25] which shows that approx. $10 \%$ of enterprises had no internet access in 2016 and more than $30 \%$ of enterprises with internet access had a data transfer rate of less than $10 \mathrm{Mbits} / \mathrm{sec}$ in 2016. Also, only $18 \%$ and $7 \%$ of the SMEs having internet access in 2015 used cloud computing services and big data analysis respectively.

The analysis also reveals that some similarities exist for different companies from different domains which is possibly due to their current state of technological levels. A certain technological feature of i4.0 can for instance be perceived as a benefit for one company and a barrier for another company. The concept of IoS may generate revenues for Company $\mathrm{E}$ but not for Company B where shifting to serviceoriented architecture may not be beneficial. The tradeoffs for i4.0 concepts appear to be related to the individual business strategies and the characteristics of the products, rather than the actual ability to implement the suggested technology.

It is also pointed out that the company culture and strategy plays an important role to adopt radical changes of the production. For example, some companies are more conservative and value having their workers there instead of replacing them with more efficient machines. The management should also be open to these changes and willing to upgrade and embrace new technologies in i4.0. Another factor is about the lack of knowledge or expertise regarding the possibility and potential of using the current technology and its applications. This has been a major problem with SMEs where more than $50 \%$ of the companies having faced difficulties to fill vacancies for IT specialists in 2016 and about $30 \%$ of companies working without their own websites [25].

\section{Conclusions}

The paper studies implementation of i4.0 concepts for SME's in the state of Brandenburg, Germany. A survey of 20 SME's revealed that companies are struggling with a range of problems in different domains. It was found that these problems are company dependent but several companies face problems related to ERP, PPS, Automation and Factory Planning.

Of the companies surveyed, five companies from different domains were considered as case studies to explore the readiness level and feasibility of implementing i4.0 concepts. An analysis of the four concepts namely IoT, IoS, CPS and smart factory pertaining to each company was performed and the potential benefits, trade-offs and barriers for implementing these concepts has been discussed.

The findings revealed that the companies are still coping with the issues relating to planning, organization and automation. It was also found that all the concepts of i4.0 may not be necessary or beneficial to an enterprise as it depends on several factors 
including their business strategies and the characteristics of the products, company culture, lack of expertise, availability of funds etc. Although i4.0 promises several benefits to the company, the infrastructure to implement these technologies is unavailable and would need considerable investments in most of the cases. It is thus concluded that the idea of i4.0 is still in its infancy and new strategies need to be developed for its realization in SME's.

\section{References}

[1] E. Westkämper, "Struktureller Wandel durch Megatrends," Digitale Produktion, pp. 7-9.

[2] World Bank. [Online] Available: https://data.worldbank.org.

[3] C. J. Bartodziej, The Concept Industry 4.0: An Empirical Analysis of Technologies and Applications in Production Logistics: Springer, 2016.

[4] H. Kagermann, J. Helbig, A. Hellinger, and W. Wahlster, Recommendations for Implementing the strategic initiative INDUSTRIE 4.0: Securing the future of German manufacturing industry; final report of the Industrie 4.0 working group: Forschungsunion, 2013.

[5] R. Drath and A. Horch, "Industrie 4.0: Hit or hype?[industry forum]," IEEE industrial electronics magazine, vol. 8, no. 2, pp. 56-58, 2014.

[6] European Commission, "2016 SBA Fact Sheet Germany," 2016. [Online] Available: http://ec.europa.eu/growth/smes/business-friendlyenvironment/performance-review-2016_en. Accessed on: Jun. 102017.

[7] L. Sommer, "Industrial revolution-industry 4.0: Are German manufacturing SMEs the first victims of this revolution?," Journal of Industrial Engineering and Management, vol. 8, no. 5, p. 1512, 2015.

[8] R. Masdefiol, M. del Mar, and F. Stävmo, Industry 4.0- Only designed to fit the German automotive industry: A multiple case study on the feasibility of Industry 4.0 to Swedish SMEs.

[9] C. Faller and D. Feldmüller, "Industry 4.0 learning factory for regional SMEs," Procedia CIRP, vol. 32, pp. 88-91, 2015.
[10] A. Decker, "Industry 4.0 and SMEs in the Northern Jutland Region," in Value Creation in International Business: Springer, 2017, pp. 309-335.

[11] M. Hermann, T. Pentek, and B. Otto, "Design principles for industrie 4.0 scenarios," in System Sciences (HICSS), 2016 49th Hawaii International Conference on, 2016, pp. 3928-3937.

[12] V. Roblek, M. Meško, and A. Krapež, "A Complex View of Industry 4.0," SAGE Open, vol. 6, no. 2, 2158244016653987, 2016.

[13] D. Giusto, A. Iera, G. Morabito, and L. Atzori, The internet of things: 20th Tyrrhenian workshop on digital communications: Springer Science \& Business Media, 2010.

[14] F. Wortmann and K. Flüchter, "Internet of Things," Bus Inf Syst Eng, vol. 57, no. 3, pp. 221-224, 2015.

[15] D. O’Halloran and E. Kvochko, "Industrial Internet of Things: Unleashing the Potential of Connected Products and Services," in World Economic Forum, 2015, p. 40.

[16] M. Brettel, N. Friederichsen, M. Keller, and M. Rosenberg, "How virtualization, decentralization and network building change the manufacturing landscape: An Industry 4.0 Perspective,"

[17] J. Gao, Y. Yao, V. C. Y. Zhu, L. Sun, and L. Lin, "Service-oriented manufacturing: A new product pattern and manufacturing paradigm," J Intell Manuf, vol. 22, no. 3, pp. 435-446, 2011.

[18] J. Cardoso, K. Voigt, and M. Winkler, "Service engineering for the internet of services," in International Conference on Enterprise Information Systems, 2008, pp. 15-27.

[19] F. Hu et al., "Robust Cyber-Physical Systems: Concept, models, and implementation," Future Generation Computer Systems, vol. 56, pp. 449-475, 2016.

[20] L. Hu, N. Xie, Z. Kuang, and K. Zhao, "Review of cyber-physical system architecture," in Object/Component/Service-Oriented Real-Time Distributed Computing Workshops (ISORCW), 2012 15 th IEEE International Symposium on, 2012, pp. 2530 . 
[21] J. Lee, B. Bagheri, and H.-A. Kao, "A CyberPhysical Systems architecture for Industry 4.0-based manufacturing systems," Manufacturing Letters, vol. 3, pp. 18-23, 2015.

[22] S. Wang, J. Wan, Di Li, and C. Zhang, "Implementing Smart Factory of Industrie 4.0: An Outlook," International Journal of Distributed Sensor Networks, vol. 12, no. 1, p. 3159805, http://dx.doi.org/10.1155/2016/3159805, 2016.
[23] J. Lee, "Smart Factory Systems," Informatik Spektrum, vol. 38, no. 3, pp. 230-235, 2015.

[24] E. Lukács and others, "The economic role of SMEs in world economy, especially in Europe," European Integration Studies, vol. 4, no. 1, pp. 3-12, 2005.

[25] Statistisches Bundesamt (Destatis), Facts \& Figures. [Online] Available: https://www.destatis.de/EN/Homepage.html. 